\title{
A distribuição do conhecimento científico público em informação, comunicação e informática em saúde indexado nas bases de dados MED LINE e LILACS
}

\author{
Public scientific knowledge distribution in health information, \\ communication and information technology \\ indexed in MEDLINE and LILACS databases
}

Abel Laerte Packer ${ }^{1}$

Adalberto O tranto Tardelli ${ }^{1}$

Regina Célia Figuei redo Castro ${ }^{1}$

${ }^{1}$ BIREM E/OPAS/OMS, Centro Latino-americano e do CaribedeInformação em Ciências da Saúde. Rua Botucatu 862, Vila

Clementino. 04023-901 São Paulo SP.

packerab@bireme.ops-oms.org

\begin{abstract}
This study explores the distribution of international, regional and national scientific output in health information and communication, indexed in the M EDLINE and LILACS databases, between 1996 and 2005. A selection of articles was based on the hierarchical structure of Information Sciencein M eSH vocabulary. Four specific domains were determined: health information, medical informatics, scientific communications on healthcare and healthcare communications. The variables analyzed were: most-covered subjects and journals, author affiliation and publication countries and languages, in both databases. The Information Science category is represented in nearly $5 \%$ of M EDLINE and LILACS articles. The four domains under analysis showed a relative annual increase in MEDLINE. The $M$ edical Informatics domain showed the highest number of records in MEDLINE, representing about half of all indexed articles. Theimportance of Information Scienceas a wholeis more visible in publications from developed countries and the findings indicate the predominance of the $U$ nited States, with significant growth in scientific output from China and South Korea and, to a lesser extent, Brazil.
\end{abstract}

Key words Information science, H ealthcare information, Medical informatics, Health Sciences communication, Health communication, Scientific production
Resumo Este artigo apresenta estudo exploratório sobre a distribuição da produção científica internacional, regional e nacional na área de informação e comunicação em saúde, referenciada nas bases de dados M ED LIN E eLILACS, de 1996 a 2005. Para a seleção dos artigos, foi usada a estrutura hierárquica do vocabulário DeCS na área de Ciência da Informação. Foram determinadosquatro domínios específicos: informação em saúde, informática médica, comunicação científica em saúde e comunicação em saúde. As variáveis analisadas foram: os assuntos e periódicos mais representativos, a produção por país de afiliação dos autores e por país de publicação e os idiomas, em ambasas bases de dados. Cerca de $5 \%$ dosartigos correspondeu à categoria Ciência da Informação. Os quatro domínios tiveram aumento relativo anual em M EDLINE. 0 domínio I nformática M édica foi o que apresentou maior número de registros em M EDLINE, representando pouco mais da metade de todos os artigos indexados. A importância da Ciência da Informação é mais visível nas publicações dos países desenvolvidos e os resultados confirmaram a predominância dos Estados U nidos e o crescimento significativo da produção científica da China e Coréia do Sul e, em menor escala, do Brasil.

Palavras-chave Ciência da informação, Informação em saúde, Informática médica, Comunicação científica em saúde, Comunicação em saúde, Produção científica 


\section{Introdução}

O conhecimento científico etécnico em saúdetem nos periódicos o seu principal meio de publicação, com controles de qualidade exercidos, entre outros, pela sua indexação em bases de dados bibliográficas. Essas bases registram, por meio de metadados de artigos científicos e outros tipos detextos (editoriais, cartas, etc.), o conhecimento público atualizado e acumulado ao longo dos anos.

Em ciências da saúde, a principal base de dados bibliográfica internacional é a MEDLINE, coordenada pela National Library of M edicine (NLM ) dos Estados Unidos, que é complementada na América Latina e Caribe pela LILACS Literatura Latino-americana e do Caribe de Informação em Ciências da Saúde, um produto cooperativo coordenado pela BIREM E/OPAS/ OMS - Centro Latino-americano e do Caribe de Informação em Ciências da Saúde, pertencenteà Organização Pan-americana da Saúde. Nestas bases de dados, estão representados os conjuntos mais relevantes da produção científica internacional e regional da América Latina e Caribe. Ambas são operadas com acesso aberto na Biblioteca Virtual em Saúde (BVS).

Além da recuperação de artigos, as bases de dados bibliográficas são fontes de informação que permitem estimar a produção científica nas diferentesáreas do conhecimento em saúde, identificar suas características e observar sua evolução ao longo dos anos nos distintos países, com base nos metadados de autores, país de afiliação institucional, título do periódico, ano de publicação, resumo e assuntos. Entretanto, as bases M EDLINE e LILACS são limitadas no registro da afiliação dos autores, assim como não incluem as referências bibliográficas recebidas pelos artigos, de modo que não é possível realizar estudos de citações, como se faz tradicionalmente com as bases do ISI, da Thomson Scientific, e mais recentemente com SciELO.

Este artigo apresenta um estudo exploratório sobrea distribuição da produção científica na área de informação, comunicação e informática em saúde, referenciada nas bases de dados M EDLINE eLILACS entre os anos 1996 e2005, situando essa produção no cenário internacional.

\section{Métodos}

0 universo de registros da produção científica internacional, regional e nacional na área de in- formação, comunicação e informática em saúde foi definido a partir de recuperação nas bases de dados M EDLINE eLILACS, disponíveis em março de 2006 na Biblioteca Virtual em Saúde, correspondente ao período de dez anos entre 1996 e 2005.

Considerou-se somente a produção científica publicada em periódicos, na forma de artigos e outras comunicações, pois a base de dados MEDLINE indexa apenas esse tipo de documentos. $\mathrm{Na}$ base LILACS, que registra todos os tipos de documentos bibliográficos, foi feita seleção dos registros correspondentes a artigos de periódicos.

\section{Fontes de informação}

A base MEDLINE é um subconjunto da base PubM ed, queindexa, além de periódicos selecionados para indexação sistemática no subconjunto M EDLINE, outros títulos que publicam esporadicamenteartigos deinteresseem ciências da saúde. Para recuperação de registros, considerou-se apenas o conjunto dos periódicos da M EDLINE, que, em dezembro de 2005, correspondia a 4.959 títulos.

A base LILACS indexava, em dezembro de 2005, 696 periódicos. Os artigos de periódicos na LILACS correspondem a $77 \%$ dos registros da base de dados.

As bases de dados são atualizadas semanalmente na BVS. 0 total de registros para o período 1996-2005 pode mudar a cada atualização, à medida que artigos publicados nessas datas sejam indexados com atraso nas bases de dados.

Os artigos indexados nas bases de dados são representados por metadados bibliográficose por descritores, que representam os assuntos discutidos, atribuídos por processo manual por profissionais da área da saúde e de ciência da informação. Os descritores são utilizados tanto na formulação de pesquisas bibliográficas como em estudos bibliométricos e cienciométricos. $\mathrm{N}$ as bases MEDLINE e LILACS, os descritores são extraídos respectivamente dos vocabulários M edical Subject H eadings ( $M$ eSH ), gerido pela N LM, e Descritores em Ciências da Saúde (DeCS), sua versão em português e espanhol, administrado pela BIREM E. Esses vocabulários foram utilizados neste estudo para a identificação dos documentos relacionados com informação, comunicação e informática em saúde.

N eles, os descritores são classificados e organizados em hierarquias, do geral para o específi- 
co, em quinze categorias temáticas, tais como: Anatomia, Organismos, Doenças, Assistência à Saúde. O DeCS tem quatro categorias adicionais criadas para a indexação da literatura da América Latina e Caribe, mas que não foram consideradas neste estudo.

A estrutura desses vocabulários é complexa e os descritores podem estar classificados em mais de uma hierarquia. Por exemplo, o descritor Linguagem aparece na hierarquia de Comunicação em duas categorias: Ciência da Informação e Psicologia e Psiquiatria, representando o meio, verbal ou não-verbal, de comunicar idéias ou sentimentos. $O$ descritor M ortalidade está incluído em sete hierarquias: na categoria de Saúde Pública, sob Demografia e sob Bioestatística; na categoria de Técnicas e Equipamentos, sob M étodos epidemiológicos; na categoria de Ciências Biológicas, novamente sob Demografia e M étodos Epidemiológicos; na categoria de Ciência da Informação, sob Coleta deDados; e na categoria de Assistência à Saúde, sob Demografia. Dependendo do número de hierarquias a que os descritores pertencem, os registros recuperados podem ser classificados em mais de uma categoria. Assim, o descritor Linguagem em um registro indica que um artigo pertencetanto à categoria de Ciência da I nformação quanto à de Psicologia e Psiquiatria. Informação adicional é necessária para resolver a ambigüidade, que pode exigir mecanismos sofisticados de recuperação.

Os descritores que caracterizam as áreas de informação, comunicação e informática em saúde estão representados na categoria L01 - Ciência da Informação. 0 primeiro nível hierárquico dessa categoria é representado pelos seguintes descritores: Colecionamento de Livros; Cronologia; Classificação; Comunicação; M eios de Comunicação; Segurança (computação); Processos de Cópia; Coleta de Dados; Centros de Informação; Gerenciamento de Informação; Serviços de Informação; Armazenamento e Recuperação da Informação; Teoria da Informação; Biblioteconomia; Informática M édica; Reconhecimento de Padrão; Editoração; Análise de Sistemas.

Deve-sedestacar que, independentemente de sua representação em uma categoria específica do DeCS, a informação e a informática têm caráter transversal e perpassam as demais categorias. De fato, todos os registros indexados nas bases de dados M EDLINE e LILACS representam em si informação ecomunicação em saúde. N este estudo, tratou-se da informação einformática em saúde como objeto da pesquisa publicada ou como aplicação de métodos e técnicas em pes- quisas de outras áreas do conhecimento em saúde. Em termos práticos, foram considerados os registros com pelo menos um descritor da categoria L01 - Ciência da Informação.

\section{Delimitação da área de informação einformática em saúde}

Para caracterizar a área de informação, comunicação e informática em saúde, foram definidos quatro subconjuntos da categoria de Ciência da Informação, denominadas neste estudo como domínios: informação em saúde, informática mé dica, comunicação científica em saúde e comunicação em saúde. Cada um destes domínios caracteriza-se pelo tipo de fonte de informação, pelas metodologias e tecnologias que são utilizadas e pelos públicos envolvidos na produção e operação dos fluxos de informação.

O domínio del nformação em Saúdeinclui artigos que estudam, utilizam, coletam ou geram informação detipo epidemiológico, demográfico de pesquisas ou censos, estatísticas vitais, e informação de suporte à vigilância em saúde, à gestão, operação e avaliação de serviços de saúde.

Informática médica (ou Informática em saúde) refere-se a artigos que fazem referência a tecnologias de informação e métodos computacionais em processos relacionados com saúde individual ecoletiva. Atualmente, o domínio foi enriquecido com o termo eH ealth, que se refere em geral à saúde e tecnologia, mas não há consenso sobre sua definição ${ }^{1}$ e o termo ainda não existe nos vocabulários M eSH e DeCS.

Comunicação Científica refere-se a artigos que tratam da produção do fluxo de informação científica, incluindo a preparação esubmissão de manuscritos, a revisão por pares, edição e publicação, documentação e indexação, sistemas de armazenamento e recuperação de informação, bases de dados, bibliometria e avaliação, bibliotecas, desenvolvimento de coleções, preservação, disseminação.

Comunicação em Saúde refere-se ao domínio dos artigos que tratam da comunicação e difusão de informação entre e para indivíduos e grupos einclui também comunicação tecnológica.

Para identificar os conceitos pertinentes a cada domínio, consideramos os 362 descritores listados na categoria de Ciência da Informação do vocabulário DeCS, num total de 449 hierarquias, separando grupos de descritores relacionados. 0 descritor Ciência da Informação, que encabeça a categoria, é definido no DeCS como 
"o campo do conhecimento, teoria, e tecnologia que lida com a coleção de fatos e números, e os processos e métodos envolvidos em sua manipulação, estocagem, disseminação, publicação e recuperação. Inclui os campos da comunicação, editoração, biblioteconomia e informática".

A identificação dos domínios constituiu uma das principais limitações deste estudo devido à ambigüidade de muitos descritores, que aparecem em mais de uma categoria ou em mais de um ramo hierárquico de uma mesma categoria. Descritores como Sistemas de informação, Disseminação de Informação, Registro e Armazenamento de Dados, Internet, para citar alguns, são representados nas hierarquias relacionadas a quase todos os domínios. M esmo em ramos hierárquicos aparentemente específicos, foram encontrados conceitos com ambigüidades, como Bases de dados bibliográficas e seus específicos (MEDLARS, MEDLINE, PUBMED, etc.) presentes em Comunicação Científica e Informática médica; ou A cervo deBiblioteca, em Comunicação Científica eM eios de Comunicação. O utras vezes, na própria definição do DeCS, o conceito corresponde a mais de um domínio, como por exemplo, Armazenamento eRecuperação da Informação é definido como "um ramo da ciência da computação ou ciência de bibliotecas relativo ao armazenamento, localização, procura e seleção [...]", isto é, se aplica tanto à Comunicação Científica quanto à Informática médica. Foi decidido manter a ambigüidade gerada pelos descritores que aparecem em mais de uma categoria.

Uma vez definidos os conjuntos de descritores de cada domínio, foram formuladas estratégias de busca, que foram aplicadas nas bases M EDLINE e LILACS, disponíveis na BVS, resultando em conjuntos específicos de registros de artigos de periódicos. Os descritores foram recuperados somente no campo de descritores primários, isto é, quando o descritor corresponde ao conceito central do documento.

A esses conjuntos foi aplicada metodologia de classificação dos descritores recuperados, por freqüência de registros na base, por relevância em relação aos descritores do domínio e por ordem hierárquica, segundo metodologia desenvolvida pela BIREME?

Para visual ização, ordenação etabulação dos registros recuperados, foi preparada uma interface própria, quepermitiu refinar as estratégias e classificar os resultados.

\section{Identificação dos países deafiliação dos autores}

A identificação do país de afiliação dos autores foi feita de maneira aproximada, devido a limitações das próprias bases de dados: a base M EDLINE, por exemplo, somente registra o país de afiliação para o primeiro autor, o que não permite identificar a afiliação dos demais autores. Desta forma, a produção medida por país referese ao primeiro autor. Além disso, o registro da afiliação écopiado do texto original do artigo e o país não tem um campo específico e normalizado, o que implica a necessidade de recorrer, em muitos casos, à pesquisa de informação adicional para identificação do país através de similaridade de textos e de regras de associação, segundo metodologia desenvolvida pela BIREM E ${ }^{3}$.

A base LILACS registra o país de todos os autores, mas passou a incluir essa informação somente em registros indexados a partir do ano 2000. Sendo assim, a informação sobre afiliação na LI LACS foi analisada a partir dessa data.

\section{Tabulação dos resultados}

Os resultados foram tabulados por:

. evolução ano a ano do total de registros recuperados em cada domínio, bem como sua representação no total dos registros indexados em cada base de dados;

- temas em destaque na produção científica de cada domínio, a partir dos descritores com maior número de registros;

- periódicos onde foram publicados artigos em cada domínio, por assunto e por país de publicação;

- países de afiliação dos autores, para comparação da produção científica brasileira, com a de países da I beroamérica (Argentina, Chile, Colômbia, Cuba, M éxico, Espanha e Portugal), países em desenvolvimento (África do Sul, China, Coréia do Sul eíndia) epaíses desenvolvidos (Estados Unidos, Inglaterra, Alemanha, Canadá, Japão e França);

- idioma das publicações.

\section{Resultados}

Em março de 2006, foram recuperados 4.906 .843 registros na baseM EDLIN E e 154.014 naLILACS, correspondentes a artigos originais e de revisão e outras comunicações como cartas, editoriais, co- 
mentários, etc. (em adiantetodos referidos como artigos), publicados entre 1996 e 2005. A partir dessa data, foram ingressados novos artigos publicados no mesmo período em ambas as bases.

A Tabela 1 apresenta a distribuição desses conjuntos de registros nas quinze categorias temáticas do DeCS. Os registros bibliográficos na base M EDLINE contêm uma média de 3,55 descritores por artigo ena LILACS, 3,18. Um artigo pode estar indexado em múltiplas categorias, devido ao uso de dois ou mais descritores de categorias diferentes ou de um mesmo descritor que pertence a duas categorias.

As áreas com maior produção científica indexada na MEDLINE são Doenças, Compostos Q uímicos e D rogas, e Ciências Biológicas, as duas primeiras presentes com pelo menos um descritor em cerca de $50 \%$ dos registros. Na LILACS, as três áreas com maior número de artigos são Doenças, Técnicas e Equipamento, e Compostos Q uímicos e Drogas, sendo que descritores da categoria D oenças aparecem em $53 \%$ dos registros. As categorias com menor número de publicações indexadas, em ambas as bases, são Locali- zações Geográficas, Humanidades, Tecnologia e Alimentos e Bebidas, relacionadas em menos de $4 \%$ dos registros.

As diferenças mais significativas entre a produção internacional indexada na M EDLINE e a regional na LILACS encontram-se nas categorias Compostos Químicos e D rogase A natomia, mais presentes na primeira nas seguintes porcentagens: $48 \%$ e $19 \%$ e $26 \%$ e $15 \%$, respectivamente. Proporcionalmente, a LI LACS contém $30 \%$ mais registros que M EDLINE nas categorias Saúde Pública e D oenças.

Os artigos relativos à informação, comunicação e informática em saúde, reunidos na categoria Ciência da Informação, objeto deste estudo, correspondem a cerca de $5 \%$ dos artigos registrados no período nas duas bases. Entretanto, a distribuição ao longo dos dez anos, assim como nos últimos anos, variam entre a MEDLINE e LILACS (Tabela 2). Enquanto na produção internacional ocorreum crescimento na participação relativa de Ciência da Informação no conjunto da MEDLINE, na LILACS é praticamente constante ao longo do período.

Tabela 1. Distribuição dos registros indexados em MEDLINE e LILACS até 2006, no período de 1996 e 2005, por categorias de assunto e ocorrência com Ciência da Informação.

\begin{tabular}{|c|c|c|c|c|c|c|c|c|c|c|}
\hline Categorias temáticas & MEDLINE & $\begin{array}{c}\% \\
M D L\end{array}$ & $\begin{array}{c}\mathrm{com} \\
\mathrm{Cl}\end{array}$ & $\begin{array}{l}\% \\
\mathrm{Cl}\end{array}$ & $\begin{array}{c}\% \text { da } \\
\text { categoria }\end{array}$ & LILACS & $\begin{array}{c}\% \\
\text { LIL }\end{array}$ & $\begin{array}{c}\mathrm{com} \\
\mathrm{Cl}\end{array}$ & $\begin{array}{l}\% \\
\mathrm{Cl}\end{array}$ & $\begin{array}{l}\% \text { da } \\
\text { categoria }\end{array}$ \\
\hline A - Anatomia & 1283864 & $26 \%$ & 24606 & $11 \%$ & $1,9 \%$ & 23370 & $15 \%$ & 318 & $4 \%$ & $1,4 \%$ \\
\hline B - Organismos & 679410 & $14 \%$ & 11570 & $5 \%$ & $1,7 \%$ & 17084 & $11 \%$ & 329 & $5 \%$ & $1,9 \%$ \\
\hline C - Doenças & 2362563 & $48 \%$ & 57807 & $25 \%$ & $2,4 \%$ & 81400 & $53 \%$ & 2354 & $33 \%$ & $2,9 \%$ \\
\hline $\begin{array}{l}\text { D - Compostos Químicos e } \\
\text { Drogas }\end{array}$ & 2330865 & $48 \%$ & 27928 & $12 \%$ & $1,2 \%$ & 29822 & $19 \%$ & 396 & $6 \%$ & $1,3 \%$ \\
\hline E - Técnicas e Equipamentos & 1385610 & $28 \%$ & 81692 & $35 \%$ & $5,9 \%$ & 49041 & $32 \%$ & 2004 & $28 \%$ & $4,1 \%$ \\
\hline F - Psicologia e Psiquiatria & 587194 & $12 \%$ & 46268 & $20 \%$ & $7,9 \%$ & 18497 & $12 \%$ & 1223 & $17 \%$ & $6,6 \%$ \\
\hline G - Ciências Biológicas & 1634955 & $33 \%$ & 82056 & $35 \%$ & $5,0 \%$ & 43408 & $28 \%$ & 2589 & $36 \%$ & $6,0 \%$ \\
\hline H - Ciências Físicas & 360092 & $7 \%$ & 42839 & $18 \%$ & $11,9 \%$ & 11220 & $7 \%$ & 1006 & $14 \%$ & $9,0 \%$ \\
\hline $\begin{array}{l}\text { I - Antropologia, Educação, } \\
\text { Sociologia e Fenômenos Sociais }\end{array}$ & 360999 & $7 \%$ & 35902 & $15 \%$ & $9,9 \%$ & 14055 & $9 \%$ & 1145 & $16 \%$ & $8,1 \%$ \\
\hline $\begin{array}{l}\text { J - Tecnologia e Alimentos e } \\
\text { Bebidas }\end{array}$ & 185041 & $4 \%$ & 8974 & $4 \%$ & $4,8 \%$ & 5086 & $3 \%$ & 203 & $3 \%$ & $4,0 \%$ \\
\hline K - Humanidades & 73697 & $2 \%$ & 7412 & $3 \%$ & $10,1 \%$ & 5406 & $4 \%$ & 442 & $6 \%$ & $8,2 \%$ \\
\hline L - Ciência da Informação & 233130 & $5 \%$ & 59570 & $26 \%$ & $25,6 \%$ & 7198 & $5 \%$ & 1632 & $23 \%$ & $22,7 \%$ \\
\hline M - Pessoas & 196090 & $4 \%$ & 16159 & $7 \%$ & $8,2 \%$ & 9084 & $6 \%$ & 726 & $10 \%$ & $8,0 \%$ \\
\hline SP - Saúde Pública & 674110 & $14 \%$ & 80354 & $34 \%$ & $11,9 \%$ & 28717 & $19 \%$ & 2412 & $34 \%$ & $8,4 \%$ \\
\hline Z - Localizações Geográficas & 783 & $0 \%$ & 61 & $0 \%$ & $7,8 \%$ & 1951 & $1 \%$ & 257 & $4 \%$ & $13,2 \%$ \\
\hline Total de registros & 4906843 & & 233130 & & & 154014 & & 7198 & & \\
\hline
\end{tabular}

MDL = M EDLINE; Cl=Ciência da Informação; LIL = LILACS 
Tabela 2. Distribuição dos registros indexados em M EDLINE e LI LACS na categoria Ciência da Informação e nos domínios Informação em saúde, Informática médica, Comunicação científica e Comunicação em saúde, por ano de publicação, entre 1996 e 2005.

\begin{tabular}{|c|c|c|c|c|c|c|c|c|c|c|c|c|}
\hline \multicolumn{13}{|c|}{ MEDLINE 1996-2005 } \\
\hline & \multicolumn{2}{|l|}{ Ano } & \multicolumn{2}{|c|}{$\begin{array}{l}\text { Ciência da } \\
\text { Informação }\end{array}$} & \multicolumn{2}{|c|}{$\begin{array}{l}\text { Informação } \\
\text { em saúde }\end{array}$} & \multicolumn{2}{|c|}{$\begin{array}{l}\text { Informática } \\
\text { em saúde }\end{array}$} & \multicolumn{2}{|c|}{$\begin{array}{l}\text { Comunicação } \\
\text { Científica }\end{array}$} & \multicolumn{2}{|c|}{$\begin{array}{l}\text { Comunicação } \\
\text { em Saúde }\end{array}$} \\
\hline & Artigos & $\begin{array}{l}\% \text { do } \\
\text { total }\end{array}$ & Artigos & $\begin{array}{l}\% \text { do } \\
\text { total } \\
\text { anual }\end{array}$ & Artigos & $\begin{array}{l}\% \text { do } \\
\text { total } \\
\text { anual }\end{array}$ & Artigos & $\begin{array}{l}\% \text { do } \\
\text { total } \\
\text { anual }\end{array}$ & Artigos & $\begin{array}{l}\% \text { do } \\
\text { total } \\
\text { anual }\end{array}$ & Artigos & $\begin{array}{l}\% \text { do } \\
\text { total } \\
\text { anual }\end{array}$ \\
\hline TOTAL & 4906843 & & 233130 & & 54479 & & 128535 & & 51391 & & 52075 & \\
\hline 1996 & 421600 & $8,6 \%$ & 16342 & $3,88 \%$ & 4433 & $1,05 \%$ & 8628 & $2,05 \%$ & 3724 & $0,88 \%$ & 3943 & $0,94 \%$ \\
\hline 1997 & 431334 & $8,8 \%$ & 18622 & $4,32 \%$ & 4861 & $1,13 \%$ & 10128 & $2,35 \%$ & 4151 & $0,96 \%$ & 4306 & $1,00 \%$ \\
\hline 1998 & 446395 & $9,1 \%$ & 19534 & $4,38 \%$ & 4900 & $1,10 \%$ & 10838 & $2,43 \%$ & 4484 & $1,00 \%$ & 4440 & $0,99 \%$ \\
\hline 1999 & 457111 & $9,3 \%$ & 20205 & $4,42 \%$ & 5205 & $1,14 \%$ & 10976 & $2,40 \%$ & 4999 & $1,09 \%$ & 4606 & $1,01 \%$ \\
\hline 2000 & 483885 & $9,9 \%$ & 21405 & $4,42 \%$ & 5134 & $1,06 \%$ & 11929 & $2,47 \%$ & 4755 & $0,98 \%$ & 4712 & $0,97 \%$ \\
\hline 2001 & 500961 & $10,2 \%$ & 22652 & $4,52 \%$ & 5552 & $1,11 \%$ & 12365 & $2,47 \%$ & 5136 & $1,03 \%$ & 4885 & $0,98 \%$ \\
\hline 2002 & 517481 & $10,5 \%$ & 23808 & $4,60 \%$ & 5216 & $1,01 \%$ & 13001 & $2,51 \%$ & 5402 & $1,04 \%$ & 5397 & $1,04 \%$ \\
\hline 2003 & 544402 & $11,1 \%$ & 27852 & $5,12 \%$ & 5993 & $1,10 \%$ & 15220 & $2,80 \%$ & 5916 & $1,09 \%$ & 6277 & $1,15 \%$ \\
\hline 2004 & 564440 & $11,5 \%$ & 31264 & $5,54 \%$ & 6602 & $1,17 \%$ & 17559 & $3,11 \%$ & 6527 & $1,16 \%$ & 6734 & $1,19 \%$ \\
\hline 2005 & 539234 & $11,0 \%$ & 31446 & $5,83 \%$ & 6583 & $1,22 \%$ & 17891 & $3,32 \%$ & 6297 & $1,17 \%$ & 6775 & $1,26 \%$ \\
\hline \multicolumn{13}{|c|}{ LILACS 1996-2005 } \\
\hline TOTAL & 154014 & & 7198 & & 3310 & & 1692 & & 1968 & & 1463 & \\
\hline 1996 & 13962 & $9,1 \%$ & 561 & $4,0 \%$ & 267 & $1,91 \%$ & 123 & $0,88 \%$ & 146 & $1,05 \%$ & 106 & $0,76 \%$ \\
\hline 1997 & 15096 & $9,8 \%$ & 757 & $5,0 \%$ & 396 & $2,62 \%$ & 173 & $1,15 \%$ & 193 & $1,28 \%$ & 112 & $0,74 \%$ \\
\hline 1998 & 15803 & $10,3 \%$ & 736 & $4,7 \%$ & 404 & $2,56 \%$ & 145 & $0,92 \%$ & 153 & $0,97 \%$ & 133 & $0,84 \%$ \\
\hline 1999 & 16359 & $10,6 \%$ & 744 & $4,5 \%$ & 351 & $2,15 \%$ & 166 & $1,01 \%$ & 188 & $1,15 \%$ & 149 & $0,91 \%$ \\
\hline 2000 & 16558 & $10,8 \%$ & 788 & $4,8 \%$ & 339 & $2,05 \%$ & 193 & $1,17 \%$ & 208 & $1,26 \%$ & 178 & $1,08 \%$ \\
\hline 2001 & 16221 & $10,5 \%$ & 775 & $4,8 \%$ & 354 & $2,18 \%$ & 188 & $1,16 \%$ & 189 & $1,17 \%$ & 152 & $0,94 \%$ \\
\hline 2002 & 16195 & $10,5 \%$ & 796 & $4,9 \%$ & 327 & $2,02 \%$ & 195 & $1,20 \%$ & 278 & $1,72 \%$ & 159 & $0,98 \%$ \\
\hline 2003 & 16849 & $10,9 \%$ & 826 & $4,9 \%$ & 356 & $2,11 \%$ & 201 & $1,19 \%$ & 226 & $1,34 \%$ & 179 & $1,06 \%$ \\
\hline 2004 & 15203 & $9,9 \%$ & 679 & $4,47 \%$ & 297 & $1,95 \%$ & 167 & $1,10 \%$ & 188 & $1,24 \%$ & 163 & $1,07 \%$ \\
\hline 2005 & 11768 & $7,6 \%$ & 536 & $4,55 \%$ & 219 & $1,86 \%$ & 141 & $1,20 \%$ & 199 & $1,69 \%$ & 132 & $1,12 \%$ \\
\hline
\end{tabular}

Fonte: bases de dados M EDLINE eLILACS, disponíveis na BVS.

Em números absolutos, entre as quinze categorias do DeCS, as mais relacionadas com Ciência da Informação são Ciências Biológicas, Técnicas e Equipamentos, Saúde Pública e Doenças, embora variem as proporções (Tabela 1). $\mathrm{Na}$ base MEDLINE, as categorias Ciências Biológicas, Técnicas e Equipamentos, e Saúde Pública aparecem associadas a 35\% dos artigos indexados em Ciência da Informação, e D oenças a $25 \%$. N a LILACS, Ciências Biológicas, Saúde Pública, e D oenças aparecem em torno de $35 \%$ dos registros e Técnicas e Equipamentos, em 28\%. Observa-se que os registros de Ciência de Informação aparecem associados a descritores de outras categorias em maior proporção que a descritores da própria categoria de Ciência da Informação, 0 que ocorreu em $26 \%$ do total na M EDLINE ena LILACS, 24\%.

Entretanto, as categorias que apresentam maior presença deartigos relacionados com Ciência da Informação são Saúde Pública e Ciências Físicas, que ocorrem em cerca de $10 \%$, ou seja, 0 dobro da presença relativa de Ciência da Informação na base de dados. $E$ as categorias $D$ oenças, Compostos Q uímicos e D rogas, A natomia e O rganismos, que têm maior presença de artigos nas bases de dados, apresentam menos de $3 \%$ dos seus registros relacionados com Ciência da Informação.

Entre os anos 1996 e2004, o número de regis- 
tros na MEDLINE aumentou 34\%, a uma taxa de crescimento média anual de 3,7\%, passando de 421.600 em 1996 a 564.440 em 2004 (Tabela 2).

$\mathrm{N}$ esse período, os artigos relacionados com Ciência da Informação aumentaram 91\%, passando de 16.342 para 31.264. Se for considerado o período de dez anos entre 1996 e 2005, a presença relativa anual dessa categoria no conjunto da M ED LIN E passou de 3,8\% em 1996 para 5,8\% em 2005. Estecrescimento éainda mais acentuado nos três anos entre 2002 e 2004, com uma taxa média de $7 \%$, enquanto a média de crescimento da base foi de $4 \%$.

Em contraste, na LILACS, o crescimento de artigos em Ciência da Informação relativo ao total da base é praticamente nulo.

Os quatro domínios de Ciência da Informação definidos no estudo tiveram aumento relativo anual de registros em relação ao total da M EDLINE, em praticamentetodos os anos entre 1996 e 2005: informação em saúde cresceu 16\%, informática médica, $62 \%$, comunicação científica, 32\% e comunicação em saúde, 34\%. Informática médica teve o crescimento mais significativo em sua representatividade na M ED LINE, principalmente a partir de 2001 , superando $10 \%$ ao ano, mais de três vezes o crescimento anual de registros na M EDLINE (Gráfico 1).
Os dez descritores que mais ocorrem na categoria de Ciência da Informação do DeCS e em cada domínio são apresentados na Tabela 3, com as respectivas frequências de ocorrência. Os resultados mostram uma distribuição consistente dos descritores por domínio. Cinco descritores ocorrem em mais deum domínio: Sistemas Computadorizados de Registros M édicos, Bases de dados Factuais, Internet, Terminologia e Redação.

0 descritor Internet tem a seguinte evolução na base de dados M EDLINE: foi criado em 1995, mas, desde 1989, 360 artigos recuperados utilizaram a palavra Internet nostítulos ou resumos de artigos. Em 1995, o descritor foi utilizado em apenas dois registros; em 1996, em 19; em 1997, em 24; apenas em 1998 passou a ser amplamente adotado pelosindexadores, com 902 artigos, que representaram 0,2\% dos registros da base e $4,6 \%$ dos registros de Ciência da Informação. A partir de 1999, a participação relativa desse descritor se mantém em torno de $0,5 \%$ no total da base, com exceção do ano 2000, quando passou a 0,6\%. $\mathrm{Na}$ área de Ciência de Informação, a participação relativa do descritor tem variado: $11,1 \%$ em 1999, $13,5 \%$ em 2000, $12,2 \%$ em 2001, 10,4\% em 2002, mantendo-se a partir daí em torno de $9 \%$.

De forma similar, pode-se observar a evolução do termo eH ealth ou e-health na base de

Gráfico 1. Distribuição dos registros da base MEDLINE indexados na categoria de Ciência da Informação, segundo agrupamentos por domínios, por ano de publicação, no período 1996-2005.

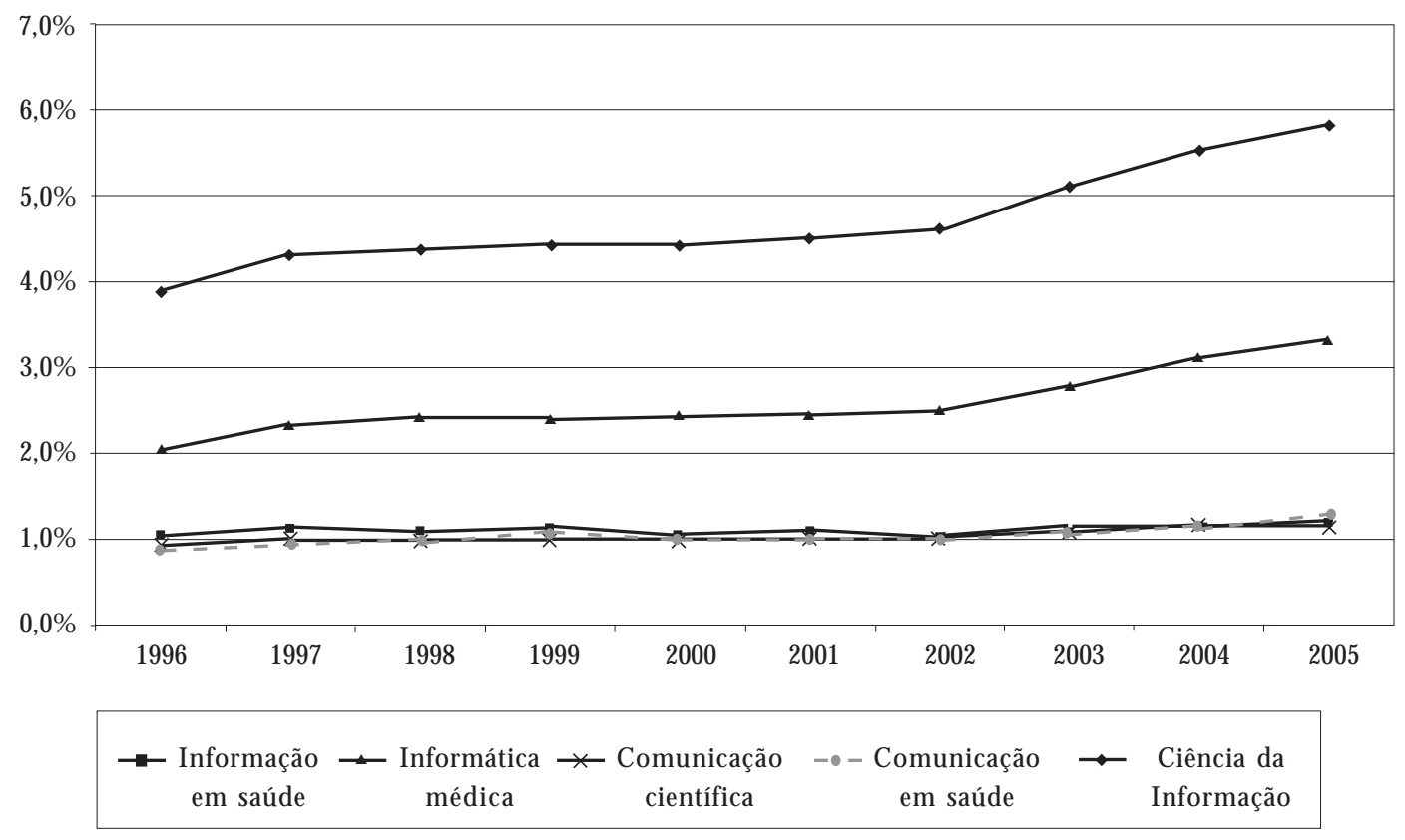


Tabela 3. Descritores mais freqüentes nos domínios de Ciência da Informação na base M EDLINE, no período 1996-2005*.

\begin{tabular}{|c|c|c|c|c|c|c|}
\hline Ciência da Informação & $\%$ & \multicolumn{2}{|c|}{ Informação em saúde } & $\%$ & Informática médica & $\%$ \\
\hline Algoritmos & 5.78 & \multicolumn{2}{|c|}{ Questionários } & 16.80 & Algoritmos & 10.49 \\
\hline $\begin{array}{l}\text { Processamento de imagem } \\
\text { assistida por computador }\end{array}$ & 4.79 & \multicolumn{2}{|c|}{$\begin{array}{l}\text { Sistemas computadorizados } \\
\text { de registros médicos }\end{array}$} & 9.89 & $\begin{array}{l}\text { Processamento de imagem } \\
\text { assistida por computador }\end{array}$ & 8.69 \\
\hline Internet & 4.59 & \multicolumn{2}{|c|}{ Bases de dados factuais } & 8.96 & Internet & 8.32 \\
\hline Questionários & 3.93 & \multicolumn{2}{|c|}{ Sistema de registros } & 7.38 & Simulação por computador & 6.34 \\
\hline Simulação por computador & 3.50 & \multicolumn{2}{|c|}{ M ortalidade } & 6.99 & Software & 5.89 \\
\hline Software & 3.25 & \multicolumn{2}{|c|}{ Serviços de informação } & 6.56 & Sistemas computadorizados & 4.19 \\
\hline Comunicação & 2.85 & \multicolumn{2}{|c|}{ Registros médicos } & 6.17 & de registros médicos & \\
\hline Publicações periódicas & 2.74 & \multicolumn{2}{|c|}{ Avaliação geriátrica } & 5.80 & Aumento da imagem & 4.10 \\
\hline Terminologia & 2.51 & \multicolumn{2}{|c|}{ Coleta de dados } & 5.51 & Imagem tridimensional & 4.08 \\
\hline Sistemas computadorizados & 2.31 & \multirow{2}{*}{\multicolumn{2}{|c|}{ Causa da morte }} & 5.36 & Bases de dados factuais & 3.80 \\
\hline de registros médicos & & & & & Redes neurais ( computação) & 3.68 \\
\hline \multicolumn{3}{|l|}{ Comunicação científica } & $\%$ & \multicolumn{2}{|c|}{ Comunicação em saúde } & $\%$ \\
\hline \multicolumn{3}{|l|}{ Publicações periódicas } & 12.43 & \multicolumn{2}{|c|}{ Comunicação } & 12.75 \\
\hline \multicolumn{3}{|l|}{ Terminologia } & 11.37 & \multicolumn{2}{|c|}{ Terminologia } & 11.22 \\
\hline \multicolumn{3}{|l|}{ Bases de dados factuais } & 9.50 & \multicolumn{2}{|c|}{ Linguagem } & 7.35 \\
\hline \multicolumn{3}{|l|}{ Editoração } & 9.49 & \multicolumn{2}{|c|}{ Leitura } & 4.65 \\
\hline \multicolumn{3}{|l|}{ Serviços de informação } & 6.96 & \multicolumn{2}{|c|}{ Semântica } & 4.65 \\
\hline \multicolumn{3}{|l|}{ Internet } & 6.20 & \multicolumn{2}{|c|}{ Publicidade } & 4.52 \\
\hline \multicolumn{3}{|c|}{ Armazenamento e recuperação da informação } & 6.08 & \multicolumn{2}{|c|}{ Fala } & 4.06 \\
\hline \multicolumn{3}{|l|}{ Sistemas de informação } & 5.18 & \multicolumn{2}{|l|}{ Redação } & 3.43 \\
\hline \multicolumn{3}{|l|}{ Redação } & 3.47 & \multicolumn{2}{|c|}{ Difusão de inovações } & 3.33 \\
\hline \multicolumn{3}{|l|}{ D ocumentação } & 3.28 & \multicolumn{2}{|c|}{ M eios de comunicação de massa } & 2.87 \\
\hline
\end{tabular}

* Frequência calculada no conjunto de registros decada domínio.

dados MEDLINE. O termo foi descrito pela primeira vez em um artigo publicado no Journal of Medical Internet Research, em 2000, que relata seu aparecimento apóso evento eH ealth Ethics Summit, realizado em Washington, DC, Estados Unidos, organizado pela Internet Healthcare Coalition, com apoio da O rganização Pan-americana da Saúde. No ano de 2000, foram recuperados 66 artigos que utilizaram esse termo nos títulos ou resumose, em 2005, 111. M as, como a freqüência de ocorrência ainda é baixa, 0 termo não foi até o momento incorporado ao vocabulário M eSH . Osdescritores mais utilizados para indexar os artigos onde aparecem os termos e-health ou eH ealth foram Internet e Telemedicina.

Considerando os assuntos utilizados para classificar os títulos de periódicos, que proporcionam uma visão mais ampla, o conjunto dos artigos indexados em Ciência da Informação fo- ram publicados em títulos de I nformática médica, M edicina, e Servi ços de saúde, abarcando 26\% do total dos registros (Tabela 4). Em cada domínio, seis ou sete assuntos de periódicos concentram 50\% dos artigos indexados. Os assuntos M edicina e Serviços de saúde estão presentes nos quatro domínios; I nformática médica eEnfermagem, em três.

No domínio de Informação em saúde, destacam-se periódi cos de Serviços desaúde, Enfermagem, Saúde pública eEpidemi ologia. Periódicos de Informática médica publicam $17 \%$ dos artigos do domínio de Informática médica, a mais alta porcentagem de freqüência de assunto de periódico registrada nos quatro domínios; em seguida, Serviços de saúde, Engenharia biomédica, Diagnóstico por imagem e Radiologia. No domínio de Comunicação científica, têm maior frequência os assuntos M edicina, Informática médica e Ciência (Tabela 4). 
Tabela 4. Distribuição dos registros de Ciência da Informação registrados na base MEDLINE por assunto dos periódicos.

\begin{tabular}{|c|c|c|}
\hline & Artigos & $\%$ \\
\hline Ciência da Informação & 233130 & \\
\hline Informática médica & 23245 & $10,0 \%$ \\
\hline M edicina & 19094 & $8,2 \%$ \\
\hline Serviços de saúde & 18884 & $8,1 \%$ \\
\hline Enfermagem & 14956 & $6,4 \%$ \\
\hline Psicologia & 9560 & $4,1 \%$ \\
\hline Neurologia & 8784 & $3,8 \%$ \\
\hline Radiologia & 8391 & $3,6 \%$ \\
\hline Diagnóstico por imagem & 8326 & $3,6 \%$ \\
\hline Engenharia biomédica & 8249 & $3,5 \%$ \\
\hline Total acumulado & & $51,3 \%$ \\
\hline Informação em saúde & 54479 & \\
\hline M edicina & 5878 & $10,8 \%$ \\
\hline Serviços de saúde & 5333 & $9,8 \%$ \\
\hline Enfermagem & 4760 & $8,7 \%$ \\
\hline Informática médica & 3977 & $7,3 \%$ \\
\hline Saúde Pública & 3770 & $6,9 \%$ \\
\hline Epidemiologia & 2464 & $4,5 \%$ \\
\hline Geriatria & 2085 & $3,8 \%$ \\
\hline Total acumulado & & $51,9 \%$ \\
\hline Informática médica & 128535 & \\
\hline Informática médica & 21965 & $17,1 \%$ \\
\hline Serviços de saúde & 12632 & $9,8 \%$ \\
\hline Engenharia biomédica & 8074 & $6,3 \%$ \\
\hline Diagnóstico por imagem & 7996 & $6,2 \%$ \\
\hline Radiologia & 7696 & $6,0 \%$ \\
\hline M edicina & 7125 & $5,5 \%$ \\
\hline Total acumulado & & $50,9 \%$ \\
\hline Comunicação científica & 51391 & \\
\hline M edicina & 6028 & $11,7 \%$ \\
\hline Informática médica & 5931 & $11,5 \%$ \\
\hline Serviços de saúde & 4708 & $9,2 \%$ \\
\hline Enfermagem & 4337 & $8,4 \%$ \\
\hline Bioquímica & 2059 & $4,0 \%$ \\
\hline Ciência & 1701 & $3,3 \%$ \\
\hline Saúde Pública & 1572 & $3,1 \%$ \\
\hline Total acumulado & & $51,2 \%$ \\
\hline Comunicação em saúde & 52075 & \\
\hline Psicologia & 6292 & $12,1 \%$ \\
\hline Enfermagem & 6201 & $11,9 \%$ \\
\hline M edicina & 5115 & $9,8 \%$ \\
\hline Serviços de saúde & 4137 & $7,9 \%$ \\
\hline Neurologia & 3138 & $6,0 \%$ \\
\hline Patologia da Fala e Linguagem & 2333 & $4,5 \%$ \\
\hline Total acumulado & & $52,3 \%$ \\
\hline
\end{tabular}

O caráter multidisciplinar dos artigos indexados na categoria de Ciência da Informação é revelado também pela quantidade de periódicos em que foram publicados. Assim, os 233.130 artigosindexados em Ciência del nformação na base de dados M EDLINE foram publicados em 5.951 periódicos e os 7.198 registros na LILACS, em 722 , que correspondem à quase totalidade de periódicos indexados nessas bases no período analisado. Os artigos de Informação em saúde foram publicados em 4.144 periódicos (70\% do total), del nformática médica, em 4.974 (84\%), de Comunicação científica, em 4.386 (74\%) e de Comuni cação em saúde, em 4.068 (68\%).

Considerando os periódicos indexados na base M EDLINE que publicaram $50 \%$ dos artigos relacionados com Ciência da Informação, foram encontrados 280 títulos diferentes no domínio de Informação em Saúde, 192 em Informática M édica, 265 em Comunicação Científica e285 em Comunicação em Saúde. Para LILACS, o número de títulos por domínio são, respectivamente, 63, 64, 48 e 46.

Ao considerar os 77 periódicos que publicaram 25\% dos artigos, observa-se que 17 (22\%) são do domínio de Informática M édica (Studies in health technology and informatics, Bioinformatics, Health management technology, Journal of telemedicine and telecare, Journal of AHIMA, Healthcare informatics, Proceedings of the American M edical Informatics Association Symposium, etc.). Entre estes 77 títulos, destacam-se os periódicos de maior cobertura científica como BM J, N ature, Science, Lancet, JAM A e Proceedings of the $\mathrm{N}$ ational A cademy of Sciences, que em conjunto respondem por 3\% da produção científica desse domínio.

$\mathrm{N}$ a base M EDLINE, foram recuperados artigos publicados em 69 periódicos classificados em Informática médica e 4 em Ciência da Informação. Na LILACS, há apenas um periódico de Informática M édica (Informática médica, da Argentina) e um de Ciência da Informação (ACIM ED, de Cuba).

Com relação à distribuição geográfica de afiliação dos autores, os artigos de Ciência da Informação registrados na base de dados MEDLINE, de 1996 a 2005, foram publicados por autores de 168 países, considerando somenteo primeiro autor. Na LILACS, foram identificados autores de 93 países, incluindo todos os autores. A distribuição total da afiliação dos autores por países é altamente concentrada, sendo que na M EDLINE autores deseis países publicaram mais de 50\% dos registros: Estados Unidos, Inglater- 
ra, Alemanha, Canadá, Japão e França, em ordem decrescente de freqüência. Em LILACS, autores do Brasil, Cuba, Argentina, Chile e Venezuela publicaram $86 \%$ do total de artigos de Ciência da Informação.

A distribuição dos artigos na área de Ciência da Informação na base M EDLINE por países de afiliação dos autores é apresentada na Tabela 5. Foram considerados os seis países com maior número de artigos, os países ibero-americanos e, para efeitos de comparação, a África do Sul, China, Coréia do Sul eĺndia. De 1996 a 2004, os seis países com maior produção registrada na base MEDLINE em Ciência da Informação permaneceram os mesmos e na mesma proporção e todos tiveram a sua participação relativa aumentada em Ciência da Informação. Por outro lado, todos tiveram a participação relativa diminuída no total da base, menos acentuada no caso do Canadá e Alemanha.

Os autores ibero-americanos publicam nos periódicos indexados na base M EDLINE menor proporção de artigos relacionados à Ciência da Informação que a outras áreas da saúde. M esmo assim, a participação relativa da América Latina aumentou significativamente entre 1996 e 2004, com uma variação positiva de $167 \%$ em Ciência da Informação, superior à taxa de crescimento de $67 \%$ relativa à base toda. 0 Brasil é o único país latino-americano que apresenta crescimento sistemático em Ciência da Informação no conjunto da MEDLINE.

A produção brasileira na base MEDLINE, medida pela afiliação do primeiro autor para todo o período, representa $0,91 \%$ detoda a basee $0,61 \%$ dos registros indexados na categoria Ciência da Informação. Em 2004, a participação relativa foi $1,24 \%$ no total da base e $0,86 \%$ em Ciência da Informação, um aumento de $160 \%$ e $133 \%$, respectivamente. Entre 2002 e 2004, 0 aumento de artigos de autores brasileiros foi de $15 \%$ na base toda e de $20 \%$ em Ciência da Informação, o que coincide com a taxa de crescimento médio mundial da categoria.

O M éxico, que é o segundo país em número de publicações científicas da América Latina e Caribe indexadas na MEDLINE, teve para todo o período de 1996 a 2005 uma participação relativa de $0,16 \%$ em Ciência da Informação, quecorresponde a cerca da metade da sua partici pação na base toda $(0,33 \%)$, com crescimento de $30 \%$ entre 1996 e 2004 no total da base e sem crescimento em Ciência da Informação. 0 terceiro país da América Latina com mais artigos, a Argentina, atingiu em 2004 uma participação relativa de
0,12\% em Ciência da Informação, inferior aos $0,30 \%$ na base toda, com crescimento respectivo de $57 \%$ e $24 \%$, aquém da média da região. 0 Chile, o quarto país com maior produção científica na América Latina e Caribe registrada na M EDLINE, apresentou o menor crescimento nesse grupo de países (Tabela 5).

A China e Coréia do Sul apresentam um crescimento extraordinário, tanto no que se refereà participação relativa em toda a base M EDLINE (188,4\% e $307,8 \%$, respectivamente) quanto na categoria Ciência da Informação, ultrapassando a cifra de $750 \%$. Contrastando, o crescimento da Índia foi de 38,7\% na basetoda e 10,8\% em Ciência da Informação. Entretanto a participação relativa em informação está ainda abaixo da média mundial de $5 \%$.

A distribuição dos artigos em Ciência da Informação na M EDLINE, tomando por baseo país de afiliação do primeiro autor e o país de publicação do periódico, revela que os autores dos Estados Unidos publicam cerca de $80 \%$ dos seus artigos em periódicos americanos. Em contraste, os autores do Canadá, França e Japão publicam, respectivamente, $16 \%, 26 \%$ e $30 \%$ em periódicos do próprio país; no total da base esses países publicam $13 \%, 28 \%$ e $30 \%$, respectivamente, em periódicos nacionais. $\mathrm{Na}$ América Latina, nos cinco principais países produtores de artigos da categoria Ciência da Informação, a porcentagem de artigos publicados nos países de origem dos autores varia consideravel mente: Argentina, 12,6\%; Brasil, 44\%; Chile, 50\%; Colômbia, $13 \%$. No total da base, a proporção de artigos publicados em periódicos nacionaisémenor para Brasil $(30 \%)$, Chile $(29 \%)$ e Colômbia ( $11 \%)$ e se mantém para Argentina. Os autores brasileiros publicaram em periódicos de 24 países, com maior porcentagem de artigos em periódicos dos Estados Unidos (26\%) elnglaterra (14\%), quejuntamente com os publicados no Brasil somam $84 \%$.

$\mathrm{Na}$ LILACS, a distribuição dos registros por país de afiliação dos autores e país de publicação revela que em média 79\% dos artigos sobre Ciência da I nformação foram publicados em periódicos nacionais; 10 países publicaram mais de $90 \%$ dos artigos em periódicos nacionais (Argentina, Bolívia, Brasil, Colômbia, Costa Rica, Cuba, H onduras, Jamaica, Porto Rico eVenezuela) e2, menos que a média: Equador (71\%) e Uruguai (52\%). Equador eU ruguai têm, respectivamente, 14 e 10 periódicos indexados na LI LACS. Os autores brasileiros publicaram $99 \%$ dos artigos de Ciência da Informação em 253 periódicos nacionais. Os periódicos brasileiros que publicaram 
Tabela 5. Distribuição dos registros da base M EDLINE por país de afiliação do primeiro autor, em relação ao total da base e à área de Ciência da Informação, 1996-2005.

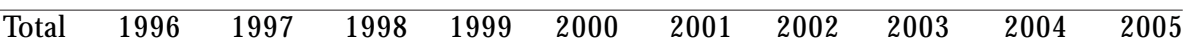

Total Ciência da Informação $233130 \quad 16342 \quad 18622 \quad 19534 \quad 20205 \quad 21405 \quad 22652 \quad 23808 \quad 27852 \quad 31264 \quad 31446$ Total MEDLINE $4906843421600431334446395457111483885500961517481544402564440 \quad 539234$

\begin{tabular}{|c|c|c|c|c|c|c|c|c|c|c|c|c|}
\hline \multicolumn{13}{|c|}{ Países - mais de $50 \%$} \\
\hline \multirow[t]{2}{*}{ Estados Unidos } & $\mathrm{Cl}$ & 74165 & 5217 & 6070 & 6085 & 6349 & 6759 & 7195 & 7562 & 9366 & 10289 & 9273 \\
\hline & MDL & 1409828 & 127767 & 130426 & 131341 & 133548 & 140415 & 144367 & 148185 & 137578 & 155327 & 160874 \\
\hline \multirow[t]{2}{*}{ Inglaterra } & $\mathrm{Cl}$ & 17622 & 1120 & 1362 & 1489 & 1542 & 1681 & 1910 & 1818 & 2207 & 2330 & 2163 \\
\hline & MD & 318727 & 27972 & 29259 & 30257 & 30540 & 32998 & 33653 & 33406 & 30500 & 34 & 35323 \\
\hline \multirow[t]{2}{*}{ Alemanha } & $\mathrm{Cl}$ & 11687 & 645 & 891 & 1003 & 887 & 1110 & 1152 & 1493 & 1413 & 1655 & 1438 \\
\hline & MDL & 249868 & 20683 & 23863 & 21928 & 23615 & 25425 & 26692 & 27513 & 24254 & 27812 & 28083 \\
\hline \multirow[t]{2}{*}{ Canadá } & $\mathrm{Cl}$ & 7782 & 442 & 552 & 627 & 629 & 678 & 740 & 866 & 1007 & 1151 & 1090 \\
\hline & $M D L$ & 146966 & 12708 & 12935 & 13346 & 13940 & 14443 & 15048 & 15496 & 16702 & 15315 & 17033 \\
\hline \multirow[t]{2}{*}{ Japão } & $\mathrm{Cl}$ & 7245 & 386 & 511 & 538 & 585 & 617 & 720 & 811 & 994 & 1125 & 958 \\
\hline & MDL & 355977 & 31670 & 32627 & 34374 & 35285 & 38066 & 37826 & 37828 & 37956 & 38125 & 32220 \\
\hline \multirow[t]{2}{*}{ França } & $\mathrm{Cl}$ & 5966 & 372 & 418 & 523 & 543 & 528 & 596 & 589 & 768 & 854 & 775 \\
\hline & MDL & 182225 & 16659 & 17317 & 17985 & 18379 & 18588 & 18818 & 18722 & 19310 & 19623 & 16824 \\
\hline \multirow[t]{2}{*}{ total - 6 países } & $\mathrm{Cl}$ & 124467 & 8182 & 9804 & 10265 & 10535 & 11373 & 12313 & 13139 & 15755 & 17404 & 15697 \\
\hline & MDL & 2663591 & 237459 & 246427 & 249231 & 255307 & 269935 & 276404 & 281150 & 266300 & 291021 & 290357 \\
\hline
\end{tabular}

Países - Ibero-américa

\begin{tabular}{|c|c|c|c|c|c|c|c|c|c|c|c|c|}
\hline Espanha & $\begin{array}{l}\mathrm{Cl} \\
\mathrm{MDL}\end{array}$ & $\begin{array}{r}3015 \\
98075\end{array}$ & $\begin{array}{r}167 \\
8011\end{array}$ & $\begin{array}{r}222 \\
8466\end{array}$ & $\begin{array}{r}209 \\
8916\end{array}$ & $\begin{array}{r}288 \\
9487\end{array}$ & $\begin{array}{r}256 \\
9758\end{array}$ & $\begin{array}{r}286 \\
10071\end{array}$ & $\begin{array}{r}321 \\
10545\end{array}$ & $\begin{array}{r}358 \\
10723\end{array}$ & $\begin{array}{r}496 \\
11756\end{array}$ & $\begin{array}{r}412 \\
10342\end{array}$ \\
\hline \multirow[t]{2}{*}{ Portugal } & $\mathrm{Cl}$ & 259 & 7 & 14 & 14 & 17 & 26 & 25 & 19 & 31 & 52 & 54 \\
\hline & MDL & 9232 & 499 & 611 & 646 & 792 & 837 & 937 & 1084 & 1224 & 1406 & 1196 \\
\hline \multirow[t]{2}{*}{ Argentina } & $\mathrm{Cl}$ & 247 & 12 & 22 & 18 & 15 & 17 & 29 & 33 & 35 & 36 & 30 \\
\hline & MDL & 14416 & 1029 & 1179 & 1215 & 1351 & 1496 & 1648 & 1747 & 1658 & 1707 & 1386 \\
\hline \multirow[t]{2}{*}{ Brasil } & $\mathrm{Cl}$ & 1422 & 54 & 56 & 89 & 92 & 120 & 142 & 171 & 228 & 269 & 201 \\
\hline & MDL & 44723 & 2249 & 2637 & 3020 & 3488 & 4107 & 4574 & 5545 & 6281 & 7015 & 5807 \\
\hline \multirow[t]{2}{*}{ Chile } & $\mathrm{Cl}$ & 146 & 10 & 16 & 8 & 11 & 7 & 11 & 26 & 17 & 24 & 16 \\
\hline & MDL & 5918 & 509 & 452 & 506 & 538 & 577 & 576 & 657 & 723 & 753 & 627 \\
\hline \multirow{2}{*}{ Colômbia } & $\mathrm{Cl}$ & 69 & 3 & 0 & 2 & 6 & 9 & 3 & 9 & 4 & 8 & 25 \\
\hline & MDL & 1821 & 84 & 96 & 100 & 134 & 171 & 176 & 265 & 253 & 285 & 257 \\
\hline \multirow[t]{2}{*}{ Cuba } & $\mathrm{Cl}$ & 71 & 3 & 3 & 5 & 7 & 5 & 6 & 7 & 6 & 16 & 13 \\
\hline & MDL & 2213 & 174 & 147 & 245 & 202 & 226 & 252 & 219 & 278 & 291 & 179 \\
\hline \multirow[t]{2}{*}{ M éxico } & $\mathrm{Cl}$ & 374 & 32 & 34 & 35 & 30 & 36 & 25 & 35 & 44 & 61 & 42 \\
\hline & MDL & 16209 & 1243 & 1329 & 1372 & 1369 & 1533 & 1647 & 1863 & 1992 & 2164 & 1697 \\
\hline \multirow[t]{2}{*}{ Outros AL\&C } & $\mathrm{Cl}$ & 231 & 13 & 10 & 15 & 14 & 29 & 27 & 34 & 16 & 40 & 33 \\
\hline & MDL & 7981 & 633 & 629 & 665 & 663 & 792 & 907 & 896 & 950 & 1015 & 831 \\
\hline \multirow[t]{2}{*}{ Total AL\&C } & $\mathrm{Cl}$ & 2560 & 127 & 141 & 172 & 175 & 223 & 243 & 315 & 350 & 454 & 360 \\
\hline & MDL & 93281 & 5921 & 6469 & 7123 & 7745 & 8902 & 9780 & 11192 & 12135 & 13230 & 10784 \\
\hline \multirow{2}{*}{$\begin{array}{l}\text { Total Ibero- } \\
\text { américa }\end{array}$} & $\mathrm{Cl}$ & 5834 & 301 & 377 & 395 & 480 & 505 & 554 & 655 & 739 & 1002 & 826 \\
\hline & $M D L$ & 200588 & 14431 & 15546 & 16685 & 18024 & 19497 & 20788 & 22821 & 24082 & 26392 & 22322 \\
\hline \multicolumn{13}{|l|}{ Outros países } \\
\hline \multirow[t]{2}{*}{ África do Sul } & $\mathrm{Cl}$ & 446 & 43 & 33 & 26 & 48 & 36 & 39 & 46 & 63 & 58 & 54 \\
\hline & MDL & 12080 & 1073 & 1107 & 1073 & 1108 & 1243 & 1314 & 1306 & 1278 & 1347 & 1231 \\
\hline \multirow[t]{2}{*}{ China } & $\mathrm{Cl}$ & 2807 & 39 & 94 & 128 & 157 & 219 & 302 & 297 & 482 & 653 & 436 \\
\hline & MDL & 98796 & 4340 & 5337 & 6447 & 7169 & 8766 & 10233 & 12514 & 15624 & 16757 & 11609 \\
\hline \multirow[t]{2}{*}{ Coréia do Sul } & $\mathrm{Cl}$ & 1011 & 14 & 35 & 39 & 49 & 76 & 100 & 107 & 143 & 231 & 217 \\
\hline & MDL & 39854 & 1248 & 1647 & 2368 & 2851 & 3682 & 4278 & 4769 & 5846 & 6813 & 6352 \\
\hline \multirow[t]{2}{*}{ Índia } & $\mathrm{Cl}$ & 999 & 75 & 67 & 86 & 47 & 86 & 89 & 98 & 132 & 159 & 160 \\
\hline & MDL & 54153 & 3869 & 3852 & 4185 & 4488 & 5149 & 5822 & 6287 & 7143 & 7185 & 6173 \\
\hline
\end{tabular}

$\mathrm{Cl}=$ Ciência da Informação; $\mathrm{MDL}=$ M EDLINE 
25\% da produção brasileira foram: Cadernos de Saúde Pública, Revista deSaúde Pública, Pro-Fono, Femina, Revista Latino-americana de Enfermagem, Revista Brasileira de Enfermagem, Revista Brasileira de Epidemiologia e Psicologia: reflexão e crítica.

Finalmente, com relação aos idiomas dos textos, na M ED LIN E predomina o inglês em $90 \%$ dos registros na categoria de Ciência da Informação, seguido do alemão $(2,6 \%)$, francês $(1,5 \%)$ e espanhol $(1,1 \%)$. Estas cifras se aproximam da distribuição na base toda: inglês ( $90 \%$ ), alemão efrancês ( $1,6 \%$ cada) eespanhol $(1,2 \%)$. Na LILACS, o inglês é menos utilizado ( $13 \%$ dos registros) e as publicações são predominantemente em espanhol (49\%) e português (40\%), o que evidencia o seu caráter regional.

\section{Discussão}

O estudo abordou a amplitude da categoria de Ciência da Informação na pesquisa científica em saúde indexada nas bases M EDLINE e LILACS, compondo quatro subconjuntos ou domínios, que na prática setraduzem em disci plinas e campos profissionais diferentes. A distribuição dos registros nestes quatros domínios revelou consistência no contexto de cada uma das bases de dados, considerando o caráter internacional da M ED LINE com predominância daprodução científica dos Estados Unidos e o caráter regional da LILACS com predominância do Brasil.

Embora as fronteiras temáticas da categoria de Ciência da Informação no M eSH possam ser criticadas, a sua estrutura de descritores permitiu identificar um conjunto de artigos e das relações com as demais categorias temáticas de saúde representadas nesse vocabulário. Em estudo similar relacionado ao domínio de Informática em Saúde, Pagliari et al. ${ }^{1}$ utilizaram o vocabulário M eSH na análise da produção científica sobre ehealth e concluíram que apenas a base MEDLINE tem uma taxonomia hierárquica de descritores que cobre essa área. No levantamento realizado em várias bases de dados, os autores concluíram que a produção dessa área se distribui entre várias áreas do conhecimento.

A Informática M édica éo domínio com maior número de registros na categoria de Ciência da I nformação na M EDLINE, representando ao Iongo dos dez anos um pouco mais da metade de todos os artigos indexados na categoria, enquanto as outras categorias participam em $22 \%$ dos registros. Esta presença marcante é ainda mais acentuada a partir de 2001, quando a representatividade dos artigos relacionados com informática médica na M EDLI NE cresceu em média o dobro do crescimento da base de dados. Este resultado evidencia o papel crescenteque as tecnologias de informação vêm desempenhando no tratamento e comunicação da informação, na automação dos processos e embutida nos equipamentos médicos. Entretanto, estedesempenho não se verifica na produção científica publicada nos periódicos da América Latina e Caribe e indexada na LILACS, onde o domínio Informação em Saúde contém quase $50 \%$ dos registros da categoria de Ciência da Informação, enquanto Informática M édica contém $25 \%$.

O caráter multidisciplinar da Ciência da Informação se revela, também, no conjunto da produção científica em saúde, pela coocorrência de seus descritores com todas as demais categorias. Este fato ocorre em ambas as bases de dados e com distribuições equivalentes, o que indica um padrão de coocorrência ou multidisciplinaridade.

M orris et al. ${ }^{4}$ discutiram a interdisciplinaridade da informática médica e como esta se relaciona com áreas correlatas, por meio da análise de periódicos e de co-citações. Os resultados mostraram que os artigos de informática médica foram recuperados em bases de dados médicas ou de ciência da informação e que os periódicos onde foram publicados se enquadravam desde "periódicos orientados à engenharia e instrumentação" até" "periódicos orientados para a prática clínica".

O caráter multidisciplinar da informação foi também constatado pelo fato que $70 \%$ ou mais dos periódicos indexados em ambas as bases de dados publicaram algum artigo que foi indexado por um descritor de Ciência da Informação. Sem muitos periódicos específicos de informática médica e ciência da informação na América Latina, como acontece em outras regiões do mundo, a utilização dos descritores do DeCS na recuperação permitiu identificar artigos relacionados aesses domínios na base de dados LILACS.

Esta dispersão vale para os quatro domínios, embora o domínio Informática M édica apresente maior consistência ao ter $17 \%$ dos artigos publicados em periódicos classificados como informática médica, seguido de periódicos em assuntos correlatos em biomedicina, tais como engenharia, radiologia e diagnóstico por imagem e serviços de saúde. Não obstante esta consistência, o domínio Informática M édica foi identificado em artigos publicados em $84 \%$ dos periódi- 
cos, a mais alta cifra entre os quatro domínios, 0 que parece indicar, por um lado, um universo de artigos de pesquisa científica em informática médica e, por outro lado, sua aplicação como tecnologia de apoio às pesquisas em outras áreas temáticas. Perry et al. ${ }^{5}$, ao analisar as fronteiras entre a informática médica e a ciência da informação em saúde, concluíram que "a ampliação da informática médica, a falta de clareza nas distinções entre os diferentes tipos de materiais, e 0 movimento das bibliotecas quanto ao uso predominante de serviços automatizados, ofereceu à ciência da informação eà informática médica a oportunidade de desenvolver relações de sinergia combinando as fortalezas de ambas disciplinas".

Os resultados deste estudo permitiram ex- plorar quantitativamente a distribuição da produção científica na área deinformação, comunicação e informática em saúde, referenciada nas bases de dados MEDLINE e LILACS, e situá-la no cenário internacional da comunicação cientifica em saúde. Seria necessário, entretanto, um estudo qualitativo dos artigos e periódicos recuperados para uma análise crítica e detal hada sobre a pesquisa científica em cada domínio.

Contudo, os resultados mostram que a importância da Ciência da Informação em toda sua amplitudeé mais visível na pesquisa cientifica em saúde dos países desenvolvidos e confirmam a predominância dosEstadosUnidoseo crescimento significativo da produção científica da China e Coréia do Sul e, em menor escala, do Brasil.

\section{Colaboradores}

AL Packer participou da concepção, análise dos resultados e redação final; AO Tardelli, da metodologia de extração de dados, análise dos resultados e redação final; RCF Castro, da preparação das tabelas, análise dos resultados e redação final.

\section{Referências}

1. Pagliari C, Sloan D, Gregor P, Sullivan F, Detmer D, Kahan JP, et al. What is eH ealth (4): a scoping exercise to map the field. J M ed Internet Res [serial on the Internet] 2005 [cited 2006 Ago 10]; 7(1):e9. Available from: http://www.jmir.org/2005/1/e9/

2. Tardelli AO, Anção MS, Packer AL, Sigulem D. Descoberta baseada em literatura: um enfoque experimental para descoberta aberta em bases de dados do tipo MEDLINE. In: VIII Congresso Brasileiro de Informática em Saúde; 2002; Natal. [acessado 2006 Ago 10]. Disponível em: http://ambienteaprendiz.bvs.br/ pdf/aot medline.pdf

3. Tardelli AO, Anção MS, Packer AL, Sigulem D. An implementation of the trigram phrase matching method for text similarity problems. Stud $\mathrm{H}$ ealth Technol Inform 2004; 103:43-9.

4. Morris TA, McCain KW. The structure of medical informatics journal literature. J Am M ed Inform Assoc 1998; 5(5):448-66.

5. Perry GJ, Roderer NK, Assar S. A current perspective on medical informatics and health sciences librarianship. J M ed Libr Assoc 2005; 93(2):199-205.

Artigo apresentado em 18/08/2006

Aprovado em 04/12/2006

Versão final apresentada em 10/12/2006 